\title{
A Control Architecture With Stabilizer For 3D Stable Dynamic Walking of SHERPA Biped Robot on Compliant Ground
}

\author{
A. Chemori, S. Le Floch, S. Krut and E. Dombre
}

\begin{abstract}
This paper deals with design and implementation of a control architecture for 3D dynamic walking with foot/ground compliant contact. This architecture includes a ZMP-based pattern generator, a computed torque controller based on the reduced order dynamics of the system, and a stabilizer to enhance the stability robustness of the control architecture. The effectiveness of the proposed control architecture is shown through numerical simulations.
\end{abstract}

\section{INTRODUCTION AND RELATED WORKS}

Legged robots are those mechanical structures which are able to move on ground by alternating support legs. The problem of control of such robots is more complex than the case of classical robot manipulators with a fixed base, inasmuch as the legs are not attached to the ground and consequently they can lift off during movement. Furthermore, control of legged robots should take into account stability of the whole structure of the robot during walking. Most successful legged robots have 2 (biped), 4 (quadrupeds) or 6 (hexapods) legs. This legs-over-wheels approach lends itself for use in all-terrain purposes seeing as legs are more effective in an uneven ground than wheels. One interesting class of legged robots is biped walking robots where mainly two sub-classes are studied, namely 2D and 3D biped robots. In biped walking locomotion, two gaits could be underlined. Static walking which refers to a system that stays balanced by always keeping the center of mass (COM) of the system vertically projected over the polygon of support formed by feet. On the contrary, dynamic walking is not constrained in such a manner, therefore the COM may leave the support polygon for periods of time.

When interesting in 3D biped walking robots, many control approaches have been proposed to resolve the problem of locomotion control. The basic control architecture in this context includes mainly three components, namely the trajectory generator, the controller and the stabilizer.

The classical way to deal with stable trajectories generation is to use the concept of Zero Moment Point (ZMP) [2] [5] [19]. However, other criteria have also been proposed, such as the Contact Wrench Sum (CWS) [9] or the Foot Rotation Indicator (FRI) [16].

The aim of the second component is to track reference trajectories, generated by the pattern generator. Within this framework, many control approaches have been proposed in the literature. A classical Proportional Derivative (PD) controller has been proposed in [8] to control HanSaRam-VII,

A. Chemori, S. Le Floch, S. Krut, and E. Dombre are with LIRMM, Univ. Montpellier 2 - CNRS, 161 rue Ada, 34392 Montpellier, France Leflochelirmm.fr a 27 degrees of freedom (dof) 3D biped walking robot. In [1], the problem of control of BIP, a 15 dof 3D biped walking robot is resolved through a nonlinear predictive control scheme with constraints. In [3], a 12 dof 3D biped walking robot has been controlled thanks to an optimal controller. In [15], an impedance control has been proposed to control a 12 dof 3D biped robot in simulation, while in [14], the same authors have proposed a hybrid control architecture based on an impedance control and computed torque control for the same application. A feedforward PID controller has been proposed in [6] to deal with walking control of Pneumat-BT, a 3D 13 dof biped robot with 26 artificial muscles. Even if these controllers are not the only ones used in 3D biped robot control, they highlight the diversity of the proposed solutions in term of control schemes.

In order to improve the performance and robustness of the controller with respect to numerical errors and external perturbations, the control architecture needs an outer control loop, this is achieved through the stabilizer. The stabilization could be performed at different levels by modifying trajectories either in joint space, in operational space, or in both. In joint space, one solution chosen to stabilize the posture of the 12 dof robot WL-10RD was to modify the ankle joints' trajectories of the support leg [20]. An other solution is that proposed in [10], where the idea was to update the hip's trajectory. In operational space, one of the original ideas, is that of Raibert [17], where the author has proposed to modify the feet's trajectories to change landing position such that the stability and the behavior of a hopping robot is improved. This idea was reproduced in [4] on P2 Honda robot, where the authors have proposed a complex control architecture including different stabilizers (body inclination control, ZMP control, and ground reaction force control) as well as the Raibert's foot landing controller.

In this paper, a control architecture (cf. figure 5) is proposed for a 3D biped walking robot nicknamed SHERPA, to deal with stable dynamic walking control. Simulation results are presented to illustrate the effectiveness of the proposed control architecture through the obtained biped walking on horizontal compliant ground.

The paper is organized as follows: In section II, the prototype of SHERPA robot is introduced as well as its different mathematical models (kinematics and dynamics), the compliant contact model is also introduced and discussed in this section. In section III, the proposed control architecture is introduced where its components (pattern generator, controller, and stabilizer) are detailed. Section IV is devoted 
to simulation results, obtained by implementation of the proposed control architecture within a simulator of SHERPA robot. The paper ends with some concluding remarks in section $\mathrm{V}$.

\section{SHERPA BIPED ROBOT}

\section{A. Robot prototype: description and dynamics}

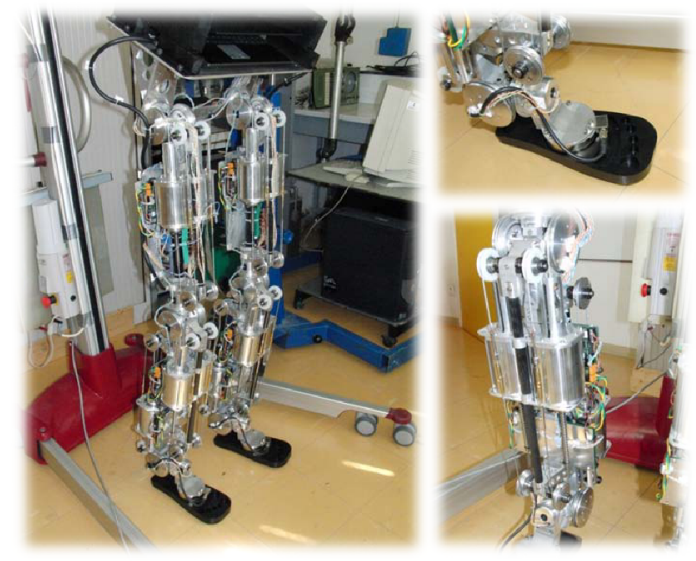

Fig. 1. Overview of SHERPA biped walking robot (left), the robot's ankle and foot (top right), the robot's tibia (bottom right).

1) Articulated mechanical structure: The main objective of a French national project named ANR-SHERPA was to design a walking robot carrying loads in a human environment. SHERPA prototype (cf. figure 1) is a $107 \mathrm{~cm}$ 3D biped robot [13] developed at LIRMM laboratory ( $w w w$. lirmm.fr). The biped robot is composed of a waist, linking two legs. Each leg has six dof, three rotations compose the spherical joint of the hip, one rotation for the knee and two rotations for the ankle. The schematic view of its articulated mechanical structure is shown in figure 3 .

These 12 dof are actuated by six actuation modules. Each module (cf. figure 2) is composed of two AC brushless motors actuating two dof using a cable based differential system.

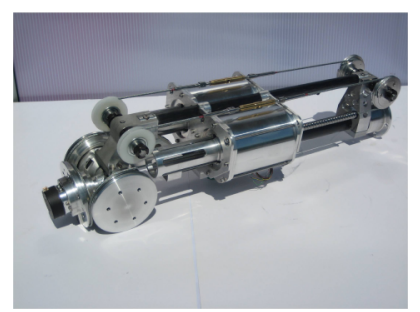

Fig. 2. Overview of one actuation module of SHERPA biped robot.

2) Kinematic models: The 12 articular positions are not enough to fully describe the robot in space, therefore the position and orientation of the robot should also be taken into account. Three translations and three rotations, virtually linking the waist to the ground reference frame, are added to the 12 dof to compose the vector of generalized coordinates. Two kinematic models have been computed. The forward kinematic model gives the 3D position of the COM and tag points (feet, knees, hips, etc) in operational space as a function of generalized coordinates in joint space $q \in \mathbb{R}^{18}$. Here, $X_{\text {tags }} \in \mathbb{R}^{45}$ is a vector containing 15 tags points coordinates. This model is described by the following equation:

$$
X_{\text {tags }}=f(q) ; q \in \mathbb{R}^{18} ; X_{\text {tags }} \in \mathbb{R}^{45}
$$

The inverse kinematic model makes the opposite transfor-

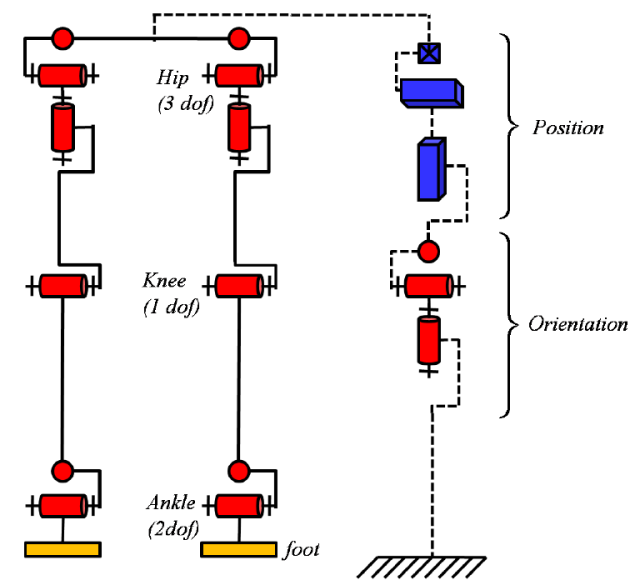

Fig. 3. Schematic view of the articulated mechanical structure of SHERPA walking robot showing its articulations and degrees of freedom.

mation between joint space and operational space:

$$
q=f^{-1}\left(X_{\text {tags }}\right) ; q \in \mathbb{R}^{18} ; X_{\text {tags }} \in \mathbb{R}^{45}
$$

3) Dynamic model: An 18 dof Lagrangian dynamic model [7] was computed and integrated in the robot simulator. This model is described by the following equation:

$$
M(q) \ddot{q}+C(q, \dot{q}) \dot{q}+G(q)=S u
$$

where $q=\left[q_{1} \ldots q_{12} \omega_{x} \omega_{y} \omega_{z} T_{x} T_{y} T_{z}\right]^{T} \in \mathbb{R}^{18}$ is the vector of generalized coordinates, $M(q) \in \mathbb{R}^{18 \times 18}$ is the inertia matrix, $C(q, \dot{q}) \in \mathbb{R}^{18 \times 18}$ is the matrix of Coriolis and centrifugal terms and $G(q) \in \mathbb{R}^{18}$ is the gravity vector. $u \in \mathbb{R}^{12}$ is the control input vector and $S \in \mathbb{R}^{18 \times 12}$ is the matrix of distribution of the torques on the articulations.

If the robot is in contact with the ground in a single or a double support phase, some efforts are applied on the feet. Then the dynamic equation becomes:

$$
M(q) \ddot{q}+C(q, \dot{q}) \dot{q}+G(q)=S u+J(q)^{T} \lambda
$$

$\lambda$ and $J(q)$ are, respectively, the Lagrangian multipliers, associated to the contact forces, and the Jacobian matrix.

\section{B. Foot/ground compliant contact model}

Since the robot is in contact with the ground, a dynamic model of the contact environment is necessary to describe the interaction between robot's rubber soles and the ground. Four contact points by foot have been considered. To simulate the force sensor that measures the contact effort a 3D model 
of compliant contact (spring-damper) is used (cf. figure 4). A linear compliant contact model along axis $\vec{z}$ could be described by:

$$
\vec{F}_{z}= \begin{cases}\left(k_{z}\left(z-z_{0}\right)-\mu_{z} \dot{z}\right) \vec{z} & \text { if } z<z_{0} \\ \overrightarrow{0} & \text { otherwise }\end{cases}
$$

Equation (5) is a well-known model of compliance, with $k_{z}$ the stiffness coefficient and $\mu_{z}$ the damping coefficient. The drawback of such a model is that at $z=z_{0}$, if $\dot{z} \neq 0, \vec{F}_{z}$ becomes discontinuous, which creates problems of physical accuracy and numerical integration of the dynamic. To deal with this problem, a nonlinear contact model should be more appropriate. The contact effort for this model is given by:

$$
\vec{F}_{z}= \begin{cases}\left(k_{z}\left|z-z_{0}\right|-\mu_{z}^{\prime}\left|z-z_{0}\right| \dot{z}\right) \vec{z} & \text { if } z<z_{0} \\ \overrightarrow{0} & \text { otherwise }\end{cases}
$$

By comparing the two contact models (5) and (6),

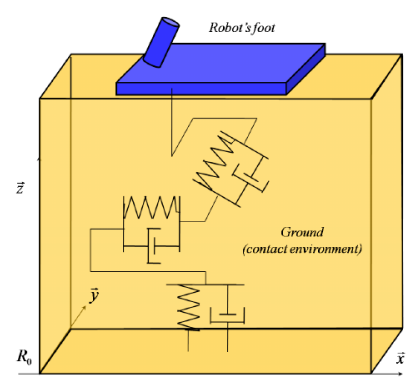

Fig. 4. 3D compliant contact based on a spring-damper model.

an equivalent damping coefficient $\mu_{z}^{\prime}|z|$ is introduced. The parameters identification process, which is easy with a linear compliant model [18], becomes empiric with this non-linear model. During the contact, the altitude $z$ remains within an interval $\left[0,-z_{\max }\right]$. In a first approach, an approximation of the damping coefficient is given by $\mu_{z}^{\prime}\left|\frac{z_{\max }}{2}\right|$. Let us now define a $3 \mathrm{D}$ compliant model by adding the tangential efforts to equation (6):

if no-sliding:

$$
\left\{\begin{array}{l}
\vec{F}_{x}=\left(k_{x}\left|x-x_{0}\right|-\mu_{x}^{\prime}\left|x-x_{0}\right| \dot{x}\right) \vec{x} \\
\vec{F}_{y}=\left(k_{y}\left|y-y_{0}\right|-\mu_{y}^{\prime}\left|y-y_{0}\right| \dot{y}\right) \vec{y}
\end{array}\right.
$$

if sliding:

$$
\left\{\begin{array}{l}
\vec{F}_{x}=-\operatorname{sign}(\dot{x}) \eta\left\|F_{z}\right\| \vec{x} \\
\vec{F}_{y}=-\operatorname{sign}(\dot{y}) \eta\left\|F_{z}\right\| \vec{y}
\end{array}\right.
$$

Where $\eta$ is the friction coefficient between foot and ground.

\section{PROPOSED CONTROL ARCHITECTURE}

This section describes the proposed control architecture used to generate stable dynamic walking. It is composed of three main components as described in figure 5. The first one is a ZMP-based pattern generator, able to generate stable walking trajectories on generalized coordinates. This pattern generator is associated with a computed torque controller to track the desired reference trajectories and a stabilizer to ensure the stability robustness of the proposed control architecture.

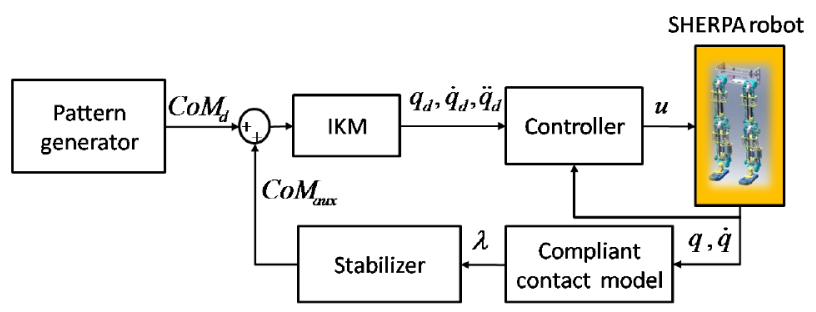

Fig. 5. Control architecture proposed for SHERPA biped robot.

\section{A. The ZMP-based pattern generator}

To generate stable walking trajectories, several pattern generators and associated criteria have been proposed in the literature. In our case, a ZMP-based pattern generator, initially proposed in [5], is considered, its basic principle is illustrated through the bloc diagram of figure 6 . Firstly,

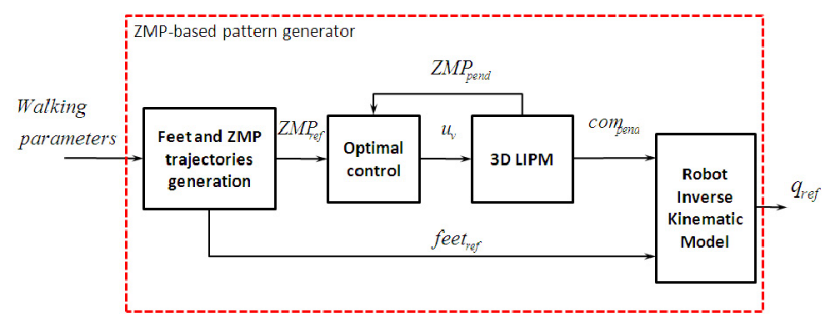

Fig. 6. ZMP based pattern generator.

ZMP and feet desired trajectories are generated by cubic interpolation. This interpolation has been made thanks to walking parameters such as step length allowing allow to determine future feet positions. Once the ZMP reference trajectory is obtained, an optimal controller is then used, with an inverted pendulum simplified model of the robot, to compute the trajectory of the COM. The optimal controller is made on a virtual actuator $u_{v}=\frac{d \ddot{x}}{d t}$, with $x$ the position of the COM. The discrete dynamic model of linear inverted pendulum is then used to obtain COM desired trajectories. Inverse kinematics model uses COM and feet trajectories to compute articulations' desired trajectories.

\section{B. Control law}

Computed torque control [7] is a well known method for a fully actuated robotic systems. Knowing the dynamic model of a system, the controller transforms the nonlinear dynamics into a linearized decoupled equivalent system.

Considering the Lagrangian dynamic model (4), SHERPA is a 18 dof system, actuated by 12 actuators. However, the robot is constrained, when it is in contact with the ground. These contact constraints allow us to reduce the order of the system's dynamics [11], [12]. 
1) Reduced order dynamic model: During walking, the biped is constantly in contact with the ground. Assuming that the friction is enough to forbid any sliding, therefore support leg do not lifts from ground during walking. For example in a single support phase, 4 contact points in space are considered. Six of these 12 geometric constraints are derived w.r.t time twice, as made in equation (11), in order to write a 6 order dynamic system using generalized coordinates.

$$
\begin{aligned}
\Phi(q) & =0 \\
\frac{\partial \Phi(q)}{\partial t} & =J(q) \dot{q}=0 \\
\frac{\partial^{2} \Phi(q)}{\partial t^{2}} & =J(q) \ddot{q}+\Pi(q, \dot{q})=0
\end{aligned}
$$

Contact constraints introduce dependencies in the vector of generalized coordinates, so that it can be split up into two vectors: actuated $q_{a}$ and unactuated $q_{n a}$ coordinates. The later can be expressed in function of the former:

$$
\begin{gathered}
q=\left[\begin{array}{cc}
q_{a}^{T} & q_{n a}^{T}
\end{array}\right]^{T} \\
J(q) \dot{q}=J_{a}(q) \dot{q}_{a}+J_{n a}(q) \dot{q}_{n a}
\end{gathered}
$$

Matrices of the reduced order model are expressed by the following [12]:

$$
\begin{aligned}
M_{*}(q) & =H^{T}(q) M(q) H(q) \\
N_{*}(q, \dot{q}) & =H^{T}(q)(N(q, \dot{q}) H(q)+M(q) \dot{H}(q))
\end{aligned}
$$

where $H(q)$ and $N(q, \dot{q})$ are given by:

$$
\begin{aligned}
H(q) & =\left(\begin{array}{c}
\mathbb{I}_{12} \\
-J_{n a}^{-1}(q) J_{a}(q)
\end{array}\right) \\
N(q, \dot{q}) & =C(q, \dot{q}) \dot{q}+G(q)
\end{aligned}
$$

So, with (4) and (11), we deduce the reduced order dynamic model equation:

$$
M_{*}(q) \ddot{q}_{a}+N_{*}(q, \dot{q})=S_{12} u
$$

In (18), $S_{12} \in \mathbb{R}^{12 \times 12}$ is given by:

$$
\begin{aligned}
S_{12} & =\operatorname{diag}\left\{S_{2}, S_{2}, S_{2}, S_{2}, S_{2}, S_{2}\right\} \\
S_{2} & =\left(\begin{array}{cc}
\frac{1}{2} & \frac{1}{2} \\
-\frac{1}{2} & \frac{1}{2}
\end{array}\right)
\end{aligned}
$$

$S_{2} \in \mathbb{R}^{2 \times 2}$ is the matrix of decomposition of the torques of one cable differential actuator module in joint space.

Remark 1: For more details about the computation of the reduced order model, the reader is referred to [11], [12].

2) Computed torques control: Once the articulation reference trajectories are computed, a control law is needed to track them in order to perform the stable walking. For that, a computed torque controller is proposed together with the reduced order model to compute the resulting closed loop system. This control must compensate all nonlinearities of the system in order to obtain a $2^{\text {nd }}$ order decoupled linear equivalent system. The proposed control law is the following:

$$
u=S_{12}^{+}\left(M_{*}(q)\left(\ddot{q}_{d}-K_{v} \dot{\tilde{q}}-K_{p} \tilde{q}\right)+N_{*}(q, \dot{q})\right)
$$

where $\tilde{q}=q_{a}-q_{d}$, the expression of $u$ in (21), replaced in (18) gives the closed-loop decoupled linear system:

$$
\ddot{\tilde{q}}+K_{v} \dot{\tilde{q}}+K_{p} \tilde{q}=0
$$

The $K_{p}$ and $K_{v}$ coefficients are the controller feedback gains. Choosing positive coefficients leads to an asymptotically stable closed loop system that is:

$$
\lim _{t \rightarrow \infty}\left(\begin{array}{c}
\tilde{q} \\
\dot{\tilde{q}}
\end{array}\right)=0
$$

With $K_{p}$ and $K_{v}$ values, it is possible to choose the closedloop dynamics as a classical $2^{\text {nd }}$ order over-damped linear system.

\section{The ZMP stabilizer}

Even if the joint trajectories are well tracked thanks to the controller, modeling errors or external disturbances could lead to the fall of the robot. Consequently, an outer stabilization loop is then needed to update online the trajectories. The aim of this stabilization is to keep the ZMP inside the polygon of support. In the pattern generator, the desired ZMP depends on COM trajectory. So, the desired COM trajectory is modified in this loop as follows. Let us the ZMP positions given by $P_{d e s}$ (the desired position), and $P_{\text {real }}$ (the real position). The ZMP position error is then given by:

$$
\Delta P=P_{\text {des }}-P_{\text {real }}
$$

To control the ZMP error $\Delta P$, for simplicity and real-time implementation reasons, a PD controller is proposed. The output of this controller is an auxiliary COM, added to the desired COM (cf. figure 5):

$$
\begin{array}{r}
x_{\text {aux }}(s)=\left(k_{p_{\text {stab }}}+s . k_{v_{\text {stab }}}\right) \Delta P \\
x_{\text {des }}=x_{\text {pend }}+x_{\text {aux }}
\end{array}
$$

The $k_{p_{s t a b}}$ and $k_{v_{s t a b}}$ coefficients have been chosen differently for single and double support phases.

\section{SIMULATION RESULTS}

The proposed control scheme has been implemented in simulation. The scenario proposed is a 14 steps with a variable (increasing) walking speed. These steps are $0.2 \mathrm{~m}$ long at a speed of $0.33 \mathrm{~m} \cdot \mathrm{s}^{-1}$. At the beginning of simulation the robot is assumed to be in rest (double support phase of $2 s$ duration). The different parameters of the walking gait, as well as the control approach are summarized in table I.

The control feedback gains $K_{p}$ and $K_{v}$ are chosen such that the resulting closed-loop dynamics behaves as a second order over-damped linear system. Stiffness and damping coefficients depend on robot weight and ground deformation at equilibrium position. With this combination, the ground deformation is of $0.5 \mathrm{~mm}$ without oscillation due to the damping. The obtained simulation results are depicted in figures 7-12.

Figure 7 shows the evolution of joint articular positions of the right leg of the robot, and figure 8 shows those of the left leg. From these two figures, it can clearly be seen the increase of walking speed (going from slow to high walking 


\begin{tabular}{|c|l|l|}
\hline Parameter & Description & Value \\
\hline $\mathrm{dt}$ & sampling time & $1 \mathrm{e}^{-2} \mathrm{sec}$ \\
\hline $\mathrm{dt} \times \mathrm{NL}$ & previewing period & $1.6 \mathrm{sec}$ \\
\hline $\mathrm{H}_{\text {pend }}$ & inverted pendulum height & $0.66 \mathrm{~m}$ \\
\hline $\mathrm{N}_{\text {pas }}$ & number of steps & $4 \mathrm{steps}$ \\
\hline $\mathrm{L}_{\text {pas }}$ & step length & $0.2 \mathrm{~m}$ \\
\hline $\mathrm{T}_{\text {pas }}$ & step duration & $0.6 \mathrm{sec}$ \\
\hline $\mathrm{K}_{p}$ & position gain & $500 \mathrm{sec}^{-2}$ \\
\hline $\mathrm{K}_{v}$ & velocity gain & $50 \mathrm{sec}^{-1}$ \\
\hline $\mathrm{k}$ & stiffness coefficient & $35 \mathrm{e}^{4} \mathrm{~N} \cdot \mathrm{m}^{-1}$ \\
\hline$\mu$ & damping coefficient & $6 \mathrm{e}^{6} \mathrm{~N} \cdot \mathrm{sec}^{2}$ \\
\hline
\end{tabular}

TABLE I

PARAMETERS USED FOR THE SIMULATION
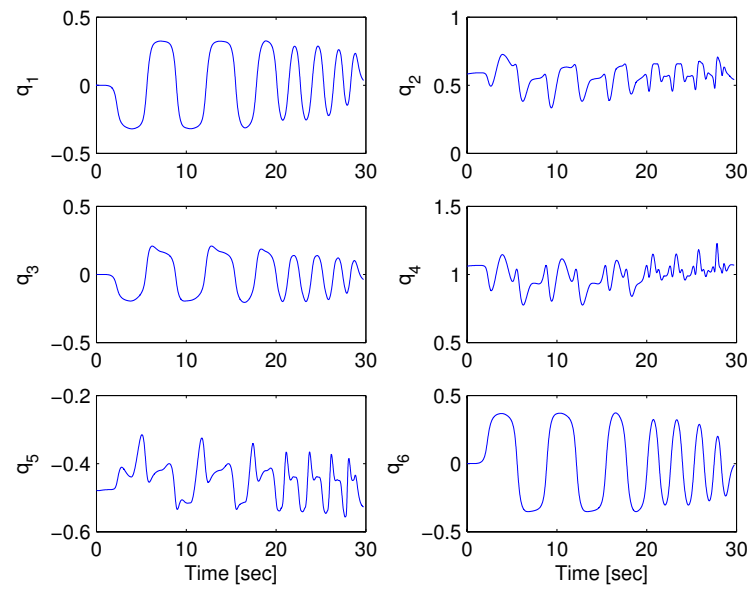

Fig. 7. Evolution of joint trajectories of the right leg versus time.

velocity).

In figure 9, The real trajectory of the robot's ZMP in the plane xoy is depicted together with the desired trajectory, as well as the associated tracking error. The extreme points correspond to the center of the robot's feet during walking. From this figure, the tracking of the $Z M P$ reference trajectory can be seen, it is quantified by the tracking error plotted at the bottom curve of figure 9 . The interaction forces at contact points of each leg with the ground are displayed in figure 10 , as well as their resultants. It can be seen from figure 10bottom that the contact forces are symmetrical with respect to each other since the robot is alternating its two legs during walking. During the single support phase, the contact force of the swing leg with the ground is equal to zero.

The evolution of the control inputs (torques generated by the proposed controller) are depicted in figures 11 for the actuator of the right leg of the robot, and on figure 12, for the left leg. One interesting remark that could be mentioned here is that the maximum torque to be generated by the actuators does not exceed $200 \mathrm{~N} . \mathrm{m}$, the maximum admissible torque that the actuators of the robot can generate. This fact shows the possibility of implementation of the proposed control architecture on the robot's prototype.
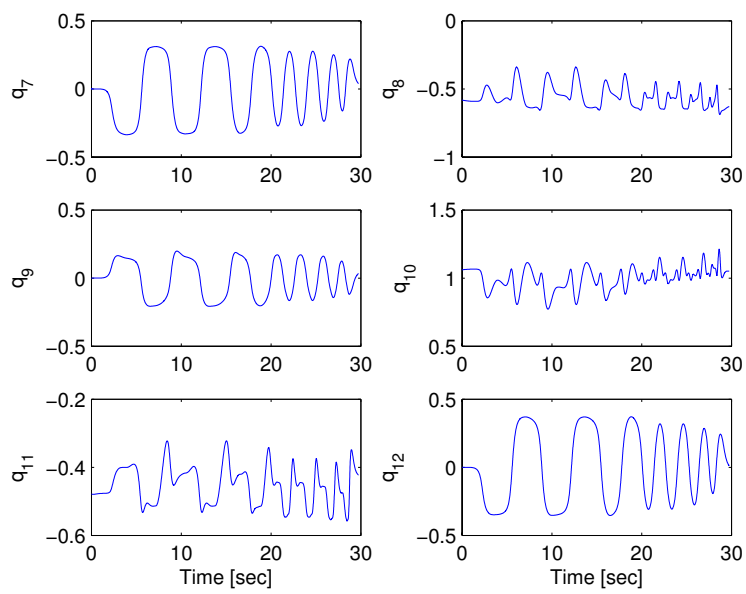

Fig. 8. Evolution of joint trajectories of the left leg versus time.
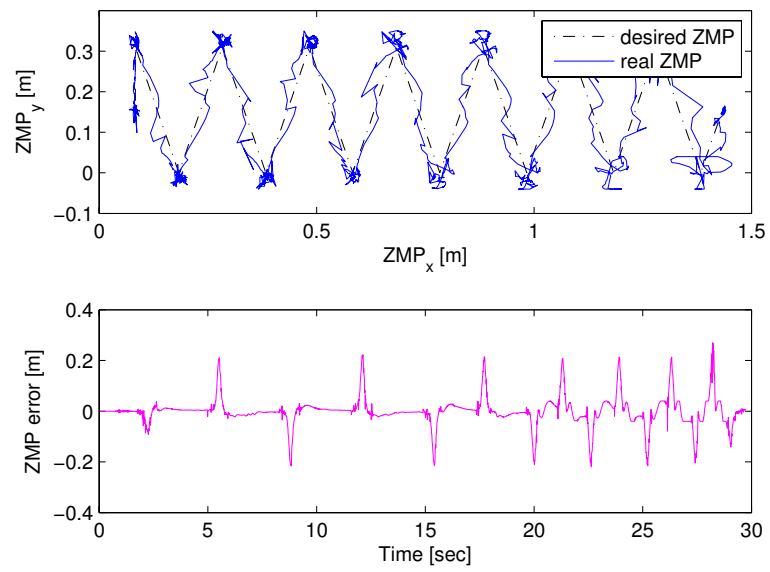

Fig. 9. Evolution of the ZMP trajectory in the plane xoy and its desired trajectory (top), evolution of the ZMP tracking error (bottom).

\section{CONCLUSION AND FUTURE WORKS}

The main objective of this paper was to propose a control architecture to simulate a $3 \mathrm{D}$ robot dynamic walking on a compliant contact environment. Dynamic models of SHERPA robot are computed a well as the dynamics of the feet/ground interaction compliant model. The proposed control architecture is composed of a ZMP based pattern generator, a computed torque controller and a stabilizer. The results are a simulation of a stable dynamic walking with increasing speed during 14 walking steps on a horizontal ground.

Our analysis leads to future works on the implementation of the proposed control architecture on the real robot's testbed. Another interesting contribution would be the study of the stabilization to modify trajectories when an unforeseen obstacle is met.

\section{ACKNOWLEDGMENTS}

This research was supported by the French National Research Agency (ANR) within the framework of the project 

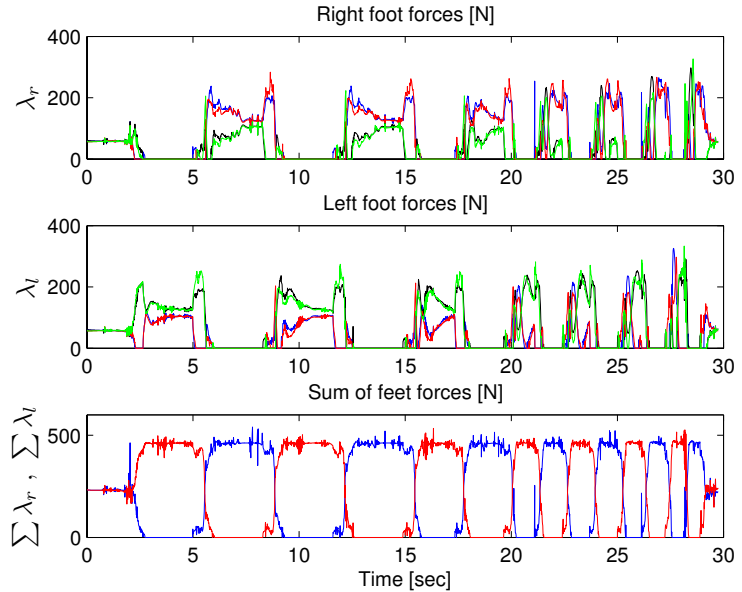

Fig. 10. Evolution of forces at contact points of the right leg (top), of the left leg (middle), and their sum per leg (bottom).
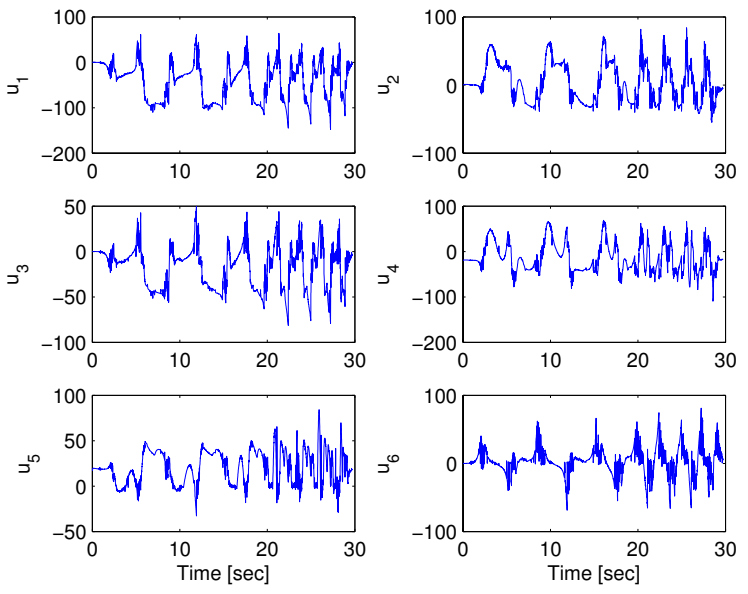

Fig. 11. Evolution of the control inputs (torques in N.m) versus time of the right leg actuators.

\section{ANR-SHERPA (ANR-06-BLAN-0244-01).}

\section{REFERENCES}

[1] C. Azevedo, P. Poignet, and B. Espiau. Artificial locomotion control: from human to robots. Robotics and Autonomous Systems (RAS), Vol. 47(4):pp.203-223, 2004.

[2] Y. Choi, D. Kim, and B.J. You. On the walking control for humanoid robot based on the kinematic resolution of com jacobian with embedded motion. In IEEE International Conference on Robotics and Automation (ICRA), Orlando, Florida, 2006.

[3] J. Denk and G. Schmidt. Synthesis of a walking primitive database for a humanoid robot using optimal control techniques. In IEEERAS International Conference on Humanoid Robots, Tokyo, Japan, November 2001.

[4] K. Hirai, M. Hirose, Y. Haikawa, and T. Takenaka. The development of honda humanoid robot. In IEEE International Conference on Robotics and Automation (ICRA), Leuven, Belgium, May 1998.

[5] H. Hirukawa, S. Kajita, F. Kanehiro, and K. Kaneko. The human-size humanoid robot that can walk, lie down and get up. The International Journal of Robotics Research, Vol.24:pp.755-769, September 2005.

[6] K. Hosoda and K. Narioka. Synergistic 3d limit cycle walking of an anthropomorphic biped robot. In IEEE/RSJ International Conference
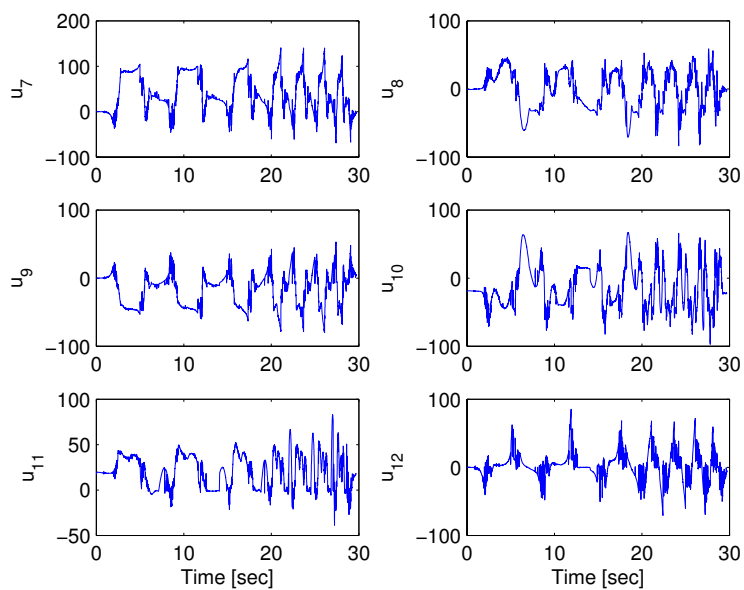

Fig. 12. Evolution of the control inputs (torques in N.m) versus time of the left leg actuators.

Intelligent Robotics Systems. (IROS), San Diego, CA, USA, October 2007.

[7] W. Khalil and E. Dombre. Modélisation identification et commande des robots. Hermes Science, 1999.

[8] Y.D. Kim, I.W. Park, J.K. Yoo, and J.H. Kim. Stabilization control for humanoid robot to walk on inclined plane. In IEEE-RAS International Conference on Humanoid Robots, Daejeon, Korea, December 2008.

[9] K. Koyanagi, H. Hirukawa, S. Hattori, M. Morisawa, and S. Nakaoka. A pattern generator of humanoid robots walking on a rough terrain using a handrail. In IEEE/RSJ International Conference Intelligent Robotics Systems. (IROS), Nice, France, September 2008.

[10] M. Kumagai and T. Emura. Attitude control of human type biped robot on a slope whose inclination is varying. In International Conference on Advanced Robotics (ICAR), Tokyo, Japan, October 1999.

[11] A. Loria and E. Panteley. Force/motion control of constrained manipulators without velocity measurements. IEEE Transactions on Automatic Control, Vol.44:pp.1407-1412, 1999.

[12] N. H. McClamroch and D. Wang. Feedback stabilization and tracking of constrained robots. IEEE Transactions on Automatic Control, Vol.33(5):pp.419-426, May 1988.

[13] I. Olaru, S. Krut, and F. Pierrot. Novel mechanical design of biped robot sherpa using 2 dof cable differential modular joints. In IEEE/RSJ International Conference Intelligent Robotics Systems. (IROS), St. Louis, MO, USA, October 2009.

[14] J. H. Park and H. Chung. Hybrid control for biped robots using impedance control and computed-torque control. In IEEE/RSJ International Conference Intelligent Robotics Systems. (IROS), Detroit, Michigan, USA, 1999.

[15] J. H. Park and H. Chung. Impedance control and modulation for stable footing in locomotion of biped robots. In IEEE/RSJ International Conference Intelligent Robotics Systems. (IROS), Takamatsu, Japan, 2000.

[16] M. B. Popovic, A. Goswami, and H. Herr. Ground reference points in legged locomotion: Definitions, biological trajectories and control implications. International Journal of Robotics Research, Vol.24(12):pp.1013-1032, 2005.

[17] M.H. Raibert. Legged robots that balance. MIT Press: Cambridge, 1986.

[18] L. Roussel. Génération de trajectoires optimales de marche pour un robot bipède. $\mathrm{PhD}$ thesis, Institut National Polytechnique de Grenoble, November 1998.

[19] P. Sardain and G. Bessonnet. Forces acting on a biped robot. center of pressurezero moment point. IEEE Transactions on Systems, Man, and Cybernetics, Vol.24(5):pp.630-637, September 2004.

[20] A. Takanishi, M. Ishida, Y. Yamazaki, and I. Kato. The realisation of dynamic walking by the biped walking robot wl-10rd. In IEEE International Conference on Robotics and Automation (ICRA), 1985. 